\title{
Evaluation of fatty proportion in fatty liver using least squares method with constraints
}

\author{
Xingsong $\mathrm{Li}^{\mathrm{a}, \mathrm{b}}$, Yinhui Deng ${ }^{\mathrm{b}}$, Jinhua $\mathrm{Yu}^{\mathrm{a}, \mathrm{c},{ }^{*},{ } \text { Yuanyuan Wang }}{ }^{\mathrm{a}}$ and Vijay Shamdasani ${ }^{\mathrm{d}}$ \\ ${ }^{a}$ Department of Electronic Engineering, Fudan University, HenglongPhysic Building, Room 212, \\ Shanghai 200433, China \\ ${ }^{b}$ Department of Healthcare, Philips Research China, Shanghai 200233, China \\ ${ }^{c}$ Key Laboratory of Medical Imaging Computing and Computer Assisted Intervention of Shanghai, \\ China \\ ${ }^{d}$ Department of Healthcare, Philips Research China, Bothell, USA
}

\begin{abstract}
Backscatter and attenuation parameters are not easily measured in clinical applications due to tissue inhomogeneity in the region of interest (ROI). A least squares method(LSM) that fits the echo signal power spectra from a ROI to a 3parameter tissue model was used to get attenuation coefficient imaging in fatty liver. Since fat's attenuation value is higher than normal liver parenchyma, a reasonable threshold was chosen to evaluate the fatty proportion in fatty liver. Experimental results using clinical data of fatty liver illustrate that the least squares method can get accurate attenuation estimates. It is proved that the attenuation values have a positive correlation with the fatty proportion, which can be used to evaluate the syndrome of fatty liver.
\end{abstract}

Keywords: Attenuation imaging, least squares method, fatty liver, quantitative ultrasound

\section{Introduction}

Fatty liver is a common phenomenon concerned in today's healthcare. It is well known that fat affects ultrasound propagation. Indeed, fat impedance is greater than that of soft tissues and hence in presence of fat, ultrasound signal will be attenuated. Currently, measurements of the mean value of ultrasound attenuation coefficient and texture features were used to distinguish fatty liver from normal liver [1-6]. Among them, the most effective available method- gray level co-occurrence matrix [7] was compared with LSM. From the result, this method can be used to diagnose fatty live by feature classification and can be used to extract the information about the location of fat, but it can't be used to evaluate fatty proportion quantitatively. To evaluate fatty proportion in fatty live and display the fat location, this paper proposes a least squares method with constraints to analyse the local attenuation value in the radio frequency (RF) signal of fatty liver. This method is applied to diagnose fatty liver by using in-vivo fatty proportion valuation. The challenge for quantitative ultrasound in this application is

${ }^{*}$ Corresponding author: Jinhua Yu, Department of Electronic Engineering, Fudan University, Shanghai, China. Tel./Fax: 021-65643202; E-mail: jhyu@fudan.edu.cn.

0959-2989/14/\$27.50 @ 2014 - IOS Press and the authors. 
related to the inhomogeneity of the liver and need of a reasonable homogeneous region to get accurate attenuation estimation.

To account for attenuation effects and backscatter effects on an inhomogeneous pathway, Kibo et al. proposed a least squares method with constraints [8] to estimate the effective attenuation between the ultrasound transducer and a ROI. This approach uses the power spectrum of RF echo signals from the same depth in a well characterized reference phantom. The ratio of the spectra fits a 3-parameter tissue model that quantifies the attenuation and backscatter properties of the media. The least squares method is introduced in detail in the next section.

In this paper, to get a correct spectral data for attenuation, the full-width-half-maximum (FWHM) [9] is used as a criterion to determine the block size. Meanwhile, one of the normal liver cases is chosen as the reference phantom. The reference phantom can be considered to have similar backscatter property as the sample phantom. Using the similarity of the backscatter coefficient as prior knowledge, the LSM can avoid solutions that are physically meaningless. Meanwhile, the uniformity of the backscatter coefficient can be considered as a criterion, the estimation is correct only when the backscatter coefficient is uniform.

\section{Least squares method with more constraints}

Based on the previous work [9], the ratio of the echo signal power spectrum from the sample to that from the reference phantom at the same depth can be expressed as:

$$
R S(f, z)=\frac{B_{s}(f)}{B_{r}(f)} \cdot \frac{A_{s}(f, z)}{A_{r}(f, z)}=\frac{b_{s} f^{n_{s}}}{b_{r} f^{n_{r}}} \exp \left(-4\left(\beta_{s}-\beta_{r}\right) f z\right)
$$

where the subscripts $s$ and $r$ represent the sample and the reference phantom, respectively. The parameter $\beta$ is the slope of the attenuation coefficient versus frequency, $b$ is a constant coefficient and $n$ expresses the frequency dependence. Taking the natural logarithm of both sides of Eq. (1), the following can be obtained:

$$
\ln \{R S(f, z)\}=\ln \frac{b_{s}}{b_{r}}+\left(n_{s}-n_{r}\right) \ln f-4\left(\beta_{s}-\beta_{r}\right) f z
$$

To simplify Eq. (2), the following terms are substituted:

$$
\begin{aligned}
& X(f, z)=\ln R S(f, z) \\
& \ln \frac{b_{s}}{b_{r}}=b, n_{s}-n_{r}=n, \beta_{s}-\beta_{r}=\beta
\end{aligned}
$$

Eq. (3) can be written as: 


$$
X(f, z)=b+n \ln f-4 \beta f z
$$

To solve for the three unknowns, $b, n$, and $\beta$ in Eq. (4), a least squares fitting process is applied over the band of frequencies contained in the echo signal. That is:

$$
[\widehat{b}, \widehat{n}, \widehat{\beta}]=\arg \min _{b, n, \beta} \sum_{i=1}^{K}\left(X\left(f_{i}, z\right)-b-n \ln f_{i}+4 \beta f_{i} z\right)^{2}
$$

where $K$ is the number of frequency components to be used for the least squares fitting and is the estimated parameters for the tissue model. Without loss of generality, Eq. (5) can be subjected to constraints to keep the result reasonable. That is:

$$
b_{1} \leq b \leq b_{2}, n_{1} \leq n \leq n_{2}, \beta_{1} \leq \beta \leq \beta_{2}
$$

With the search range of each parameter set according to expected ranges for the tissue or sample media within the ROI.

Since LSM is sensitive to the constraints, the method will converge to local minimum and lead to error. To avoid this situation, a reference phantom which has a similar backscatter property to the unknown phantom was chosen. Using the backscatter coefficients of the reference phantom as a prior knowledge, additional constraints were created as follow:

$$
b_{1} \leq b \leq b_{2}, n_{1} \leq n \leq n_{2}, \quad \beta_{1} \leq \beta \leq \beta_{2},\left|b_{1}-b_{2}\right| \leq \text { threshold } 1,\left|n_{1}-n_{2}\right| \leq \text { threshold } 2
$$

With the improved constraints, the LSM converges to the right solution more effectively.

Realistic bounds can be easily made for the range of backscatter coefficients, attenuation coefficients, and frequency dependencies of backscatter allowed for the sample, as discussed below. Once $b$, $n$, and $\beta$ are estimated, the backscatter function and effective attenuation of the sample are computed using the known values for the reference phantom and Eq. (3).

\section{Experimental result}

\subsection{Phantoms with attenuation contrast}

The least squares method was evaluated first by recording echo signal data for two tissue mimicking phantoms. One with uniform attenuation and backscatter was used as a reference (see Figure 1(a)) and the other with inhomogeneous attenuation and uniform backscatter was used as the unknown sample (see Figure 1(b)). Both phantoms were simulated by the Field II [10]. The excitation frequency of the echo data is $6 \mathrm{MHz}$ and the linear array transducer has 192 elements and 500 micron element pitch. The sampling frequency is $40 \mathrm{MHz}$. The unknown phantom has three layers with equivalent backscatter coefficient and different attenuation coefficient. The first layer ranges from $20 \mathrm{~mm}$ to $33 \mathrm{~mm}$ and its 
attenuation value is $0.3 \mathrm{~dB} / \mathrm{cm} / \mathrm{MHz}$. The second layer ranges from $33 \mathrm{~mm}$ to $47 \mathrm{~mm}$ and its attenuation value is $0.6 \mathrm{~dB} / \mathrm{cm} / \mathrm{MHz}$. The third layer ranges from $47 \mathrm{~mm}$ to $60 \mathrm{~mm}$ and its attenuation value is $1 \mathrm{~dB} / \mathrm{cm} / \mathrm{MHz}$.

After acquiring RF data, the RF data frame was divided into smaller overlapping 2-D blocks of sufficient size to obtain a stable power spectrum. A criteria based on the evaluation of the full-width-halfmaximum (FWHM) of the power spectrum was used to determine the block size that would provide a consistent power spectrum. The block power spectrum was calculated using Welch method [11] and the gated window size was set to be half the axial length of the block with an $80 \%$ window overlap used to calculate the power spectrum. Each windowed RF segment was gated by a Hanning window to minimize spectral leakage artifacts. Each block contained 10 beam lines in the lateral direction. An $80 \%$ overlap of the 2-D blocks was applied to obtain a map of the slope of attenuation coefficients. Frequency smoothing where adjacent frequency estimates were averaged using a moving average window [12], was also utilized to further reduce spectral noise artifacts in the power spectra.

The numerical phantoms were $40 \mathrm{~mm}$ wide, $100 \mathrm{~mm}$ deep and $10 \mathrm{~mm}$ thick and the axial transmit focus as well as the elevational focus were set as $40 \mathrm{~mm}$ and $70 \mathrm{~mm}$, respectively. After computing the FWHMs of the power spectra for various block sizes, the 2-D block size [13] for the computation of the power spectra was selected as $4 \times 4 \mathrm{~mm}$ along the axial and lateral dimensions respectively. The constraints used for numerical phantom are set as follows:

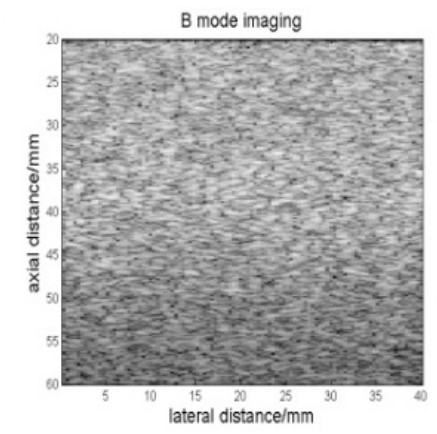

(a)

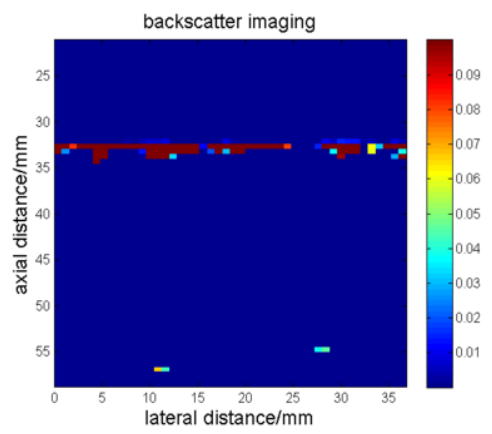

(d)

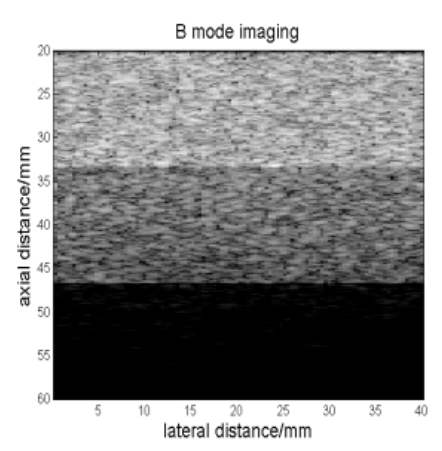

(b)

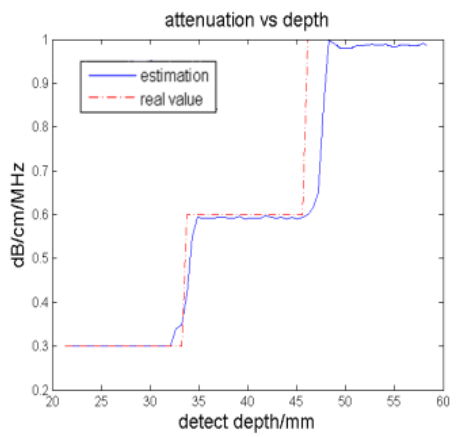

(e)

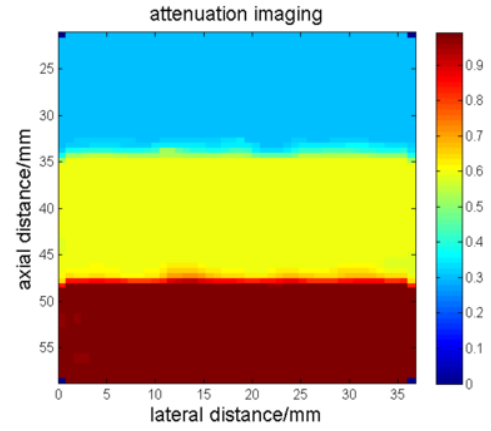

(c)

Fig. 1. Attenuation estimation in numerical phantom. (a) reference phantom (b) unknown phantom (c) attenuation imaging (d) backscatter imaging (e) attenuation value change with depth. 


$$
-1 \leq b \leq 1,0 \leq n \leq 5,-0.5 \leq \beta \leq 0.5
$$

The frequency range applied in Eq. (5) varied with depth so as to only include the frequency components that were at least $20 \mathrm{~dB}$ above the noise floor.

The attenuation image (see Figure 1(c)) was obtained by the improved LSM. Due to the reason that the backscatter coefficient in reference phantom is the same as the backscatter coefficient in unknown phantom, according to Eq. (3), $b$ is equal to 0, as the backscatter image (see Figure 1(d)) shows that the estimation value coincides with the theoretical value. To observe the performance of this method, one line in attenuation image is compared with the real value (see Figure 1(e)).It can be seen that the proposed method can provide accurate estimations of the attenuation and backscatter coefficients.

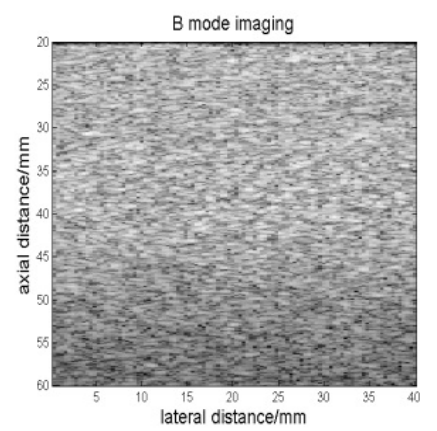

(a)

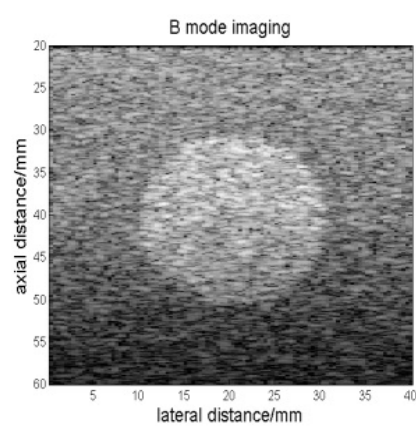

(b)

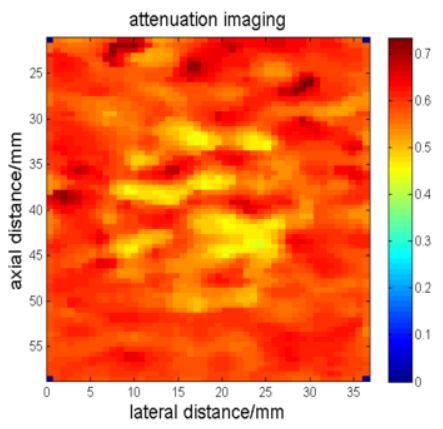

(c)

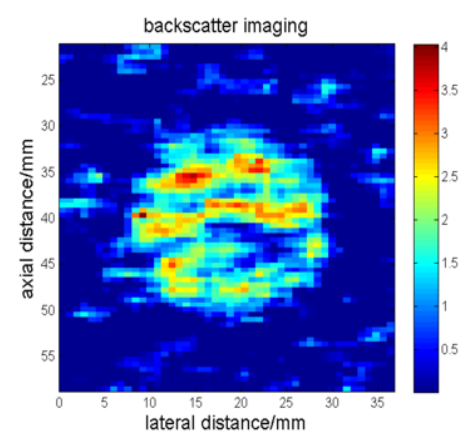

(d)

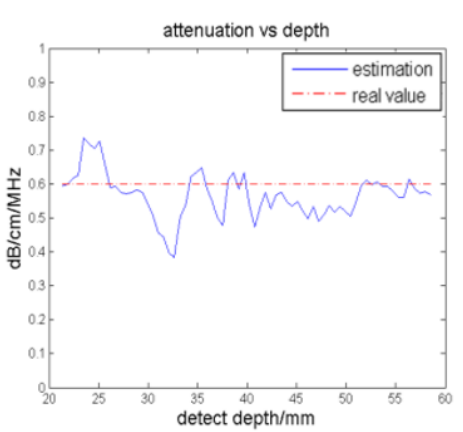

(e)

Fig. 2. Attenuation estimation in numerical phantom. (a) reference phantom (b) unknown phantom (c) attenuation image (d) backscatter image (e) attenuation value changes with depth.

Table 1

Estimation results on synthetic phantoms

\begin{tabular}{llll}
\hline Phantom Number & Mean value & Std value & Real value \\
\hline Phantoms with attenuation contrast & $0.293,0.591,0.992$ & $0.021,0.023,0.022$ & $0.3,0.6,1$ \\
Phantoms with backscatter contrast & 0.5829 & 0.0528 & 0.6 \\
\hline
\end{tabular}




\subsection{Phantoms with backscatter contrast}

The LSM to a numerical phantom with spatial variations in backscatter was also applied to test whether the method is sensitive to variations in backscatter.

The reference phantom has uniform backscatter and attenuation and its attenuation value is $0.3 \mathrm{~dB} / \mathrm{cm} / \mathrm{MHz}$ (see Figure 2(a)). The unknown phantom (see Figure 2(b)) has uniform attenuation and inhomogeneous backscatter. The backscatter levels of the inclusion were different compared with the background by a factor of $3 \mathrm{~dB}$. The unknown phantom's attenuation value is $0.6 \mathrm{~dB} / \mathrm{cm} / \mathrm{MHz}$. As shown in Figures 2(c)-2(e) and Table 1, the improved LSM also gets more accurate estimation values of the attenuation and backscatter coefficients [9].

\subsection{Tissue mimicking phantom}

Two tissue mimicking phantoms were used to test the performance of the improved LSM, and the reference phantom (see Figure 3(a)) has the similar backscatter property to the unknown phantom (see Figure 3(b)), with the attenuation value of the former being $0.5 \mathrm{~dB} / \mathrm{cm} / \mathrm{MHz}$, and that of the latter being $0.7 \mathrm{~dB} / \mathrm{cm} / \mathrm{MHz}$.

The tissue mimicking(TM) phantoms were scanned using an iU22 Philips ultrasound system equipped with a L9-3 (192 elements, 3 rows, 200 micron element pitch) linear array transducer.

Local attenuation values were estimated by the LSM and the proposed method (see Figures 3(c) and 3(d)), respectively. From the results (see Table 2), a significant improvement was observed by using the modified LSM.

\subsection{Clinical data acquisition and analysis}

To evaluate the fatty proportion of the fatty liver, normal liver and fatty liver were scanned using an

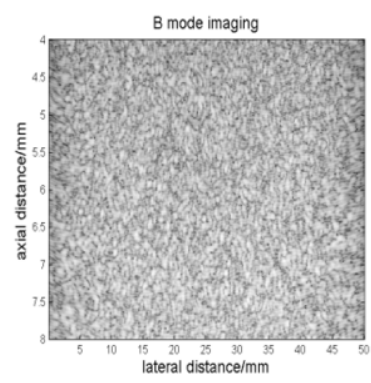

(a)

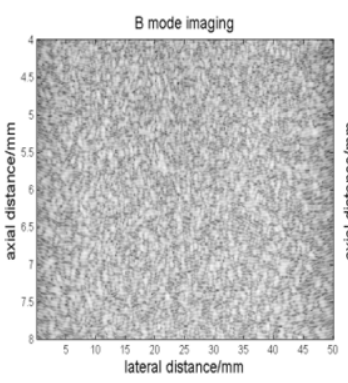

(b)

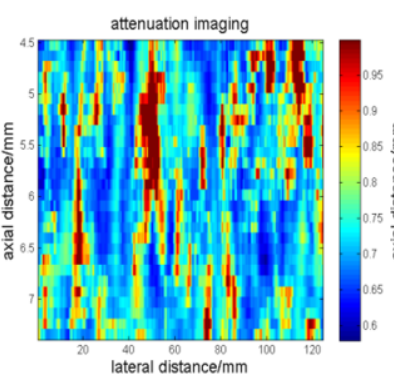

(c)

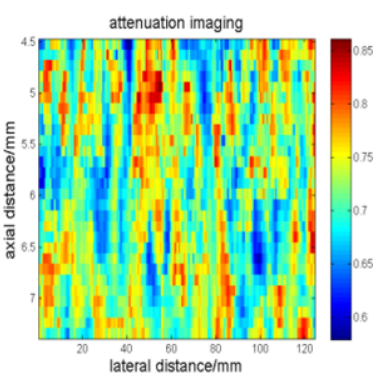

(d)

Fig. 3. Attenuation estimation in TM phantom. (a) reference phantom (b) unknown phantom (c) attenuation image with the LSM (d) attenuation image with the proposed method.

Table 2

Estimation results on TM phantoms

\begin{tabular}{llll}
\hline method & Mean value & Std value & Real value \\
\hline LSM & 0.7526 & 0.0897 & 0.7 \\
Proposed method & 0.7207 & 0.0446 & 0.7 \\
\hline
\end{tabular}


iU22 Philips ultrasound system. The ultrasound research interface on the iU22 system was used to acquire frames of RF data at a $32 \mathrm{MHz}$ sampling frequency. Data were collected at ultrasound department in Western China hospital with patients' consent and there were 16 cases of normal liver data and 11 cases of fatty liver data. A ROI from each data which has a relative uniform backscatter property was chosen. One of the normal liver data (see Figure 4(a)) was chosen as reference phantom and the attenuation values are considered to be constant at $0.59 \mathrm{~dB} / \mathrm{cm} / \mathrm{MHz}$, measured by narrow band substitution techniques [14]. With similar backscatter property, the estimation result will be more accurate. Meanwhile, the backscatter coefficient can also be used as a criterion to evaluate the reliability of the estimation, when the backscatter coefficients are uniform, the result is considered to be tenable.

One case of normal liver's local attenuation values (see Figure 4(b)) was estimated by the modified LSM. The attenuation image (see Figure 4(c)) and the backscatter image (see Figure 4(d)) were obtained by this method. The result shows that the normal liver has a relative uniform attenuation value and its mean value is $0.51 \mathrm{~dB} / \mathrm{cm} / \mathrm{MHz}$.

Meanwhile, local attenuation values of two fatty liver cases were estimated by the improved LSM. The fatty liver with $40 \%$ fatty proportion (see Figure 5(a)) has different attenuation values from $15 \%$ fatty proportion (see Figure 5(b)).

The attenuation images (see Figures 5(c) and 5(d)) show that the attenuation changes with different fatty proportions.

The data of 16 cases of normal liver and 11 cases of fatty liver were used as the unknown phantom. The mean and the standard derivation of the attenuation values are listed (see Tables 3 and 4).

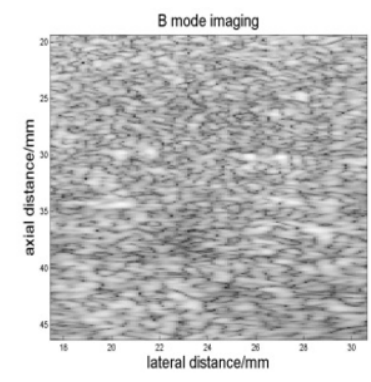

(a)

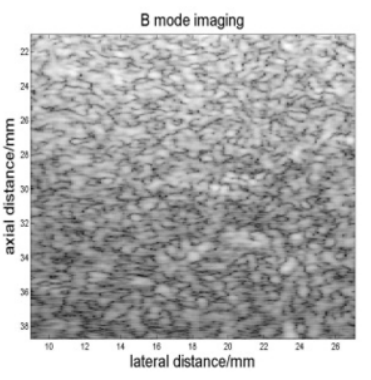

(b)

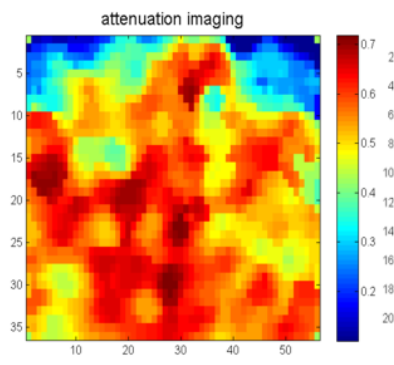

(c)

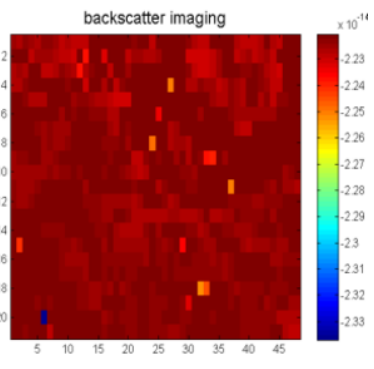

(d)

Fig. 4. Attenuation estimation in normal liver. (a) reference phantom (b) normal liver (c) attenuation imaging (d) backscatter imaging.

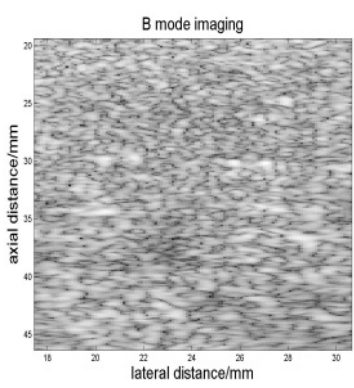

(a)

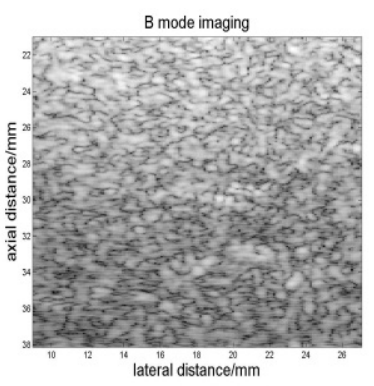

(b)

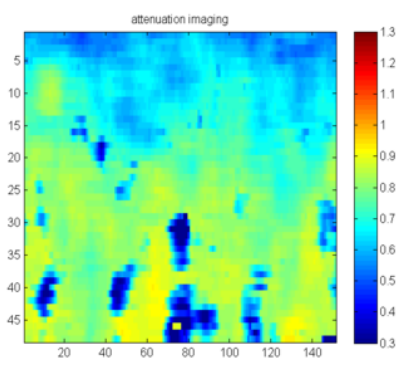

(c)

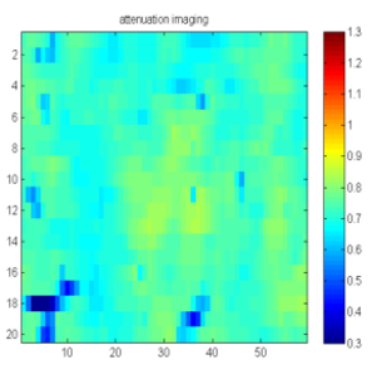

(d)

Fig. 5. Attenuation estimation in fatty liver. (a) $40 \%$ fatty liver (b) $15 \%$ fatty liver (c) attenuation image of $40 \%$ fatty liver (d) attenuation image of $15 \%$ fatty liver. 
Table 3

Estimation result of normal liver

\begin{tabular}{lllllll}
\hline Liver case & Mean value & std & & Liver case & Mean value & std \\
\hline 1 & 0.573 & 0.24 & & 9 & 0.63 & 0.15 \\
2 & 0.56 & 0.14 & & 10 & 0.62 & 0.10 \\
3 & 0.52 & 0.13 & & 11 & 0.57 & 0.136 \\
4 & 0.536 & 0.09 & & 12 & 0.67 & 0.11 \\
5 & 0.57 & 0.14 & & 13 & 0.545 & 0.132 \\
6 & 0.499 & 0.12 & & 14 & 0.66 & 0.108 \\
7 & 0.52 & 0.10 & & 15 & 0.67 & 0.09 \\
8 & 0.59 & 0.09 & 16 & 0.486 & 0.127 \\
\hline
\end{tabular}

Table 4

Estimation result of fatty liver

\begin{tabular}{lllllll}
\hline Liver case & Mean value & std & & Liver case & Mean value & std \\
\hline 1 & 0.7715 & 0.0995 & & 7 & 0.7176 & 0.1005 \\
2 & 0.7483 & 0.0463 & & 8 & 0.8847 & 0.0737 \\
3 & 0.7785 & 0.0672 & & 9 & 0.7349 & 0.0794 \\
4 & 0.5640 & 0.1046 & & 10 & 0.5730 & 0.0712 \\
5 & 0.7748 & 0.1209 & & 11 & 0.6526 & 0.0557 \\
6 & 0.6840 & 0.1294 & & & \\
\hline
\end{tabular}

To search the relationship between the fatty proportion and the attenuation value, six fatty liver cases with different fatty proportions are analyzed. Box plots of attenuation values of these six cases are shown in Figure 6. From the result, an obvious increasing trend in attenuation value was observed with the increasing fatty proportion. Due to the fact that the fatty liver's attenuation is higher than that of the normal liver [10], a reasonable threshold is chosen to search the relationship between fatty proportion and attenuation value. Here a $0.7-0.9 \mathrm{~dB} / \mathrm{cm} / \mathrm{MHz}$ attenuation value range was performed, using an increment of $0.001 \mathrm{~dB} / \mathrm{cm} / \mathrm{MHz}$. The result (see Figure 7) shows that there exist an approximately positive correlation between fatty proportion and attenuation value proportion. The mean value between the fatty liver and the normal liver was also compared as shown in Figure 8. It is shown that the attenuation coefficient values of normal liver were $0.55 \pm 0.1 \mathrm{~dB} / \mathrm{MHz} / \mathrm{cm}$, and $0.74 \pm 0.1 \mathrm{~dB} / \mathrm{MHz} / \mathrm{cm}$ in fatty liver. With a certain threshold, the mean value can also be used to detect fatty liver from normal liver.

\section{Discussion and conclusion}

In this paper, an effective attenuation coefficient of fatty liver and normal liver is measured by applying the LSM to echo data derived from a clinical scanner equipped with a research interface. To get an accurate estimation, one of the normal liver data is chosen as the reference phantom. The reference phantom is considered to have similar backscatter property to the sample phantom. Using the equality of the backscatter coefficient as prior knowledge, the LSM can avoid solutions that are physically meaningless. Meanwhile, the uniformity of the backscatter coefficient can be considered as criteria, the estimation is correct only when the backscatter coefficient is uniform. 


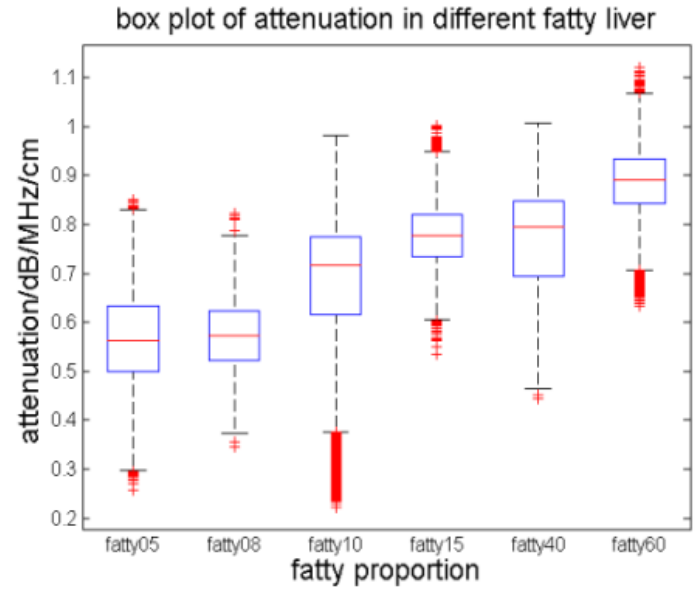

Fig. 6. The box plot of attenuation in different fatty liver.

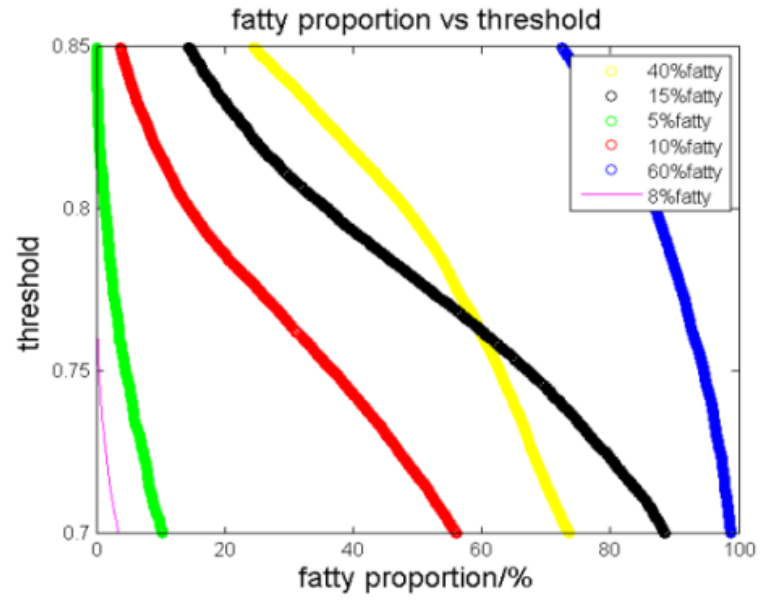

Fig. 7. The relationship between fatty proportion and attenuation value.

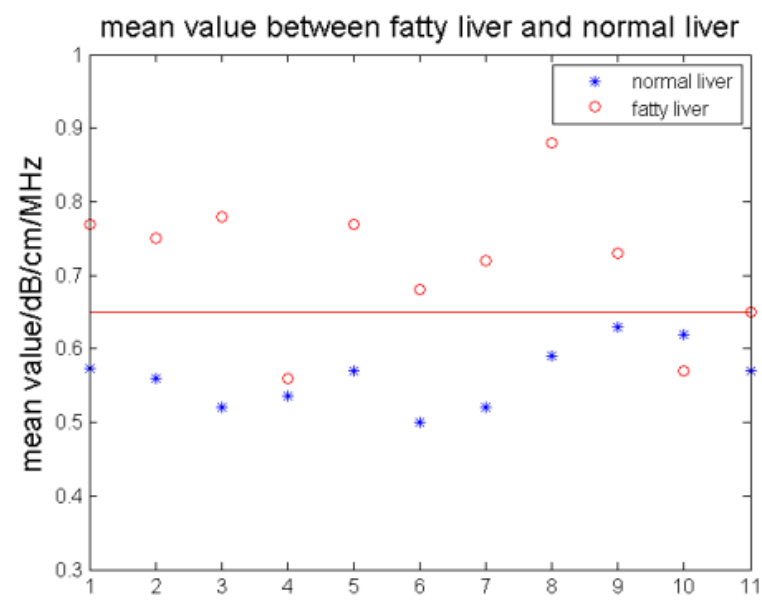

Fig. 8. Mean value between fatty liver and normal liver.

The result shows that when a tenable threshold is chosen, there exists an approximately positive correlation between fatty proportion and attenuation value proportion. With this method, attenuation coefficient values are $0.55 \pm 0.1 \mathrm{~dB} / \mathrm{MHz} / \mathrm{cm}$ in normal liver, $0.77 \pm 0.1 \mathrm{~dB} / \mathrm{MHz} / \mathrm{cm}$ in fatty liver. LSM can also be used to detect fatty liver from normal liver.

However, due to the simple functional forms for attenuation and backscatter, error will occur when these assumptions are not well met, which lead to an approximately positive correlation between fatty proportion and attenuation value proportion. One solution might be applying a piecewise continuous frequency range.

The least squares method provides accurate measures in fatty liver and normal liver as well as the backscatter coefficient vs. frequency. Meanwhile, using the least squares method, a positive correlation between fatty proportion and attenuation value can be observed. 


\section{Acknowledgment}

The assistance of Philips who provides the clinical data of normal liver and fatty liver used in this study is gratefully acknowledged. This work is supported by the National Natural Science Foundation of China (81101049 and 61271071), Shanghai Pujiang Talent Program (12PJ1401200), and Doctoral Fund of Ministry of Education (20110071120019).

\section{References}

[1] J.C. Xu, L. Sun, Y.P. Gao and T.H. Xu, An ensemble feature selection technique for cancer recognition, Bio-Medical Materials and Engineering 24 (2013), 1001-1006.

[2] S. Gao, Y.H. Peng, H.Z. Guo, W.F. Liu and T.X. Gao, Texture analysis and classification of ultrasound liver images, Bio-Medical Materials and Engineering 24 (2013), 1209-1216.

[3] S. Magali, M. Veronique and S. Laurent, Novel controlled attenuation parameter for the evaluation of fatty liver disease, IEEE International Ultrasonics Symposium Proceeding 21 (2009), 2256-2258.

[4] Y. Fujii, N. Taniguchi, K. Itoh and K. Wang, A new method for attenuation coefficient measurement in the liver: Comparison with the spectral shift central frequency method, J. Ultrasound Med. 21 (2002), 783-788.

[5] P.A. Narayana and J. Ophir, On the frequency dependence of attenuation in normal and fatty liver, IEEE Trans. Son. Ultrason. 30 (1983), 379-383.

[6] Y. Tadashi, I. Kenta, Y. KENJI and Z. Satoki, Acoustic characteristics of fatty and fibrotic liver measured by an 80MHz and $250 \mathrm{MHz}$ scanning acoustic microscopy, Joint UFFC, EFTF and PFM Symposium 1 (2013), 393-395.

[7] X.D. Wang, Y. Fang, B. Hu and H.Q. Cao, B-scan image feature extraction of fatty liver, Sixth International Conference on Internet Computing for Science and Engineering 3 (2012), 188-191.

[8] K. Nam, J.A. Zagzebski and T.J. Hall, Simultaneous backscatter and attenuation estimation using a least squares method with constraints, Ultrasound Med. Biol. 37 (2011), 2096-2104.

[9] K. Hyungsuk and T. Varghese, Hybrid spectral domain method for attenuation slope estimation, Ultrasound in Med. \& Biol. 34 (2008), 1820-1823.

[10] J.J. Field, A program for simulating ultrasound systems, The 10th Nordic-Baltic Conference on Biomedical Imaging Published in Medical \& Biomedical Engineering \& Computing 34 (1996), 351-353.

[11] P. Welch, The use of fast fourier transform for the estimation of power spectra: A method based on time averaging over short, modified periodograms, IEEE Trans. Audio Electroacoustic 15 (1967), 70-73.

[12] T. Varghese and K.D. Donohue, Estimating mean scatterer spacing with the frequency-smoothed spectral autocorrelation function, IEEE Trans. Ultrason Ferroelectr. Freq. Control 42 (1995), 451-463.

[13] L. Yassin, Optimization and application of ultrasound attenuation estimation algorithms, Ph.D. Dissertation, University of Iowa State, 2010.

[14] E.L. Madsen, G.R. Frank, P.L. Carson and P.D. Edmonds, Inter-laboratory comparison of ultrasonic attenuation and speed measurements, J. Ultrasound Med. 5 (1986), 569-576. 